\title{
Is There an Association between Urolithiasis and Roux-En-Y Gastric Bypass Surgery?
}

\author{
Andre Costa-Matos, Luiz R. Guidoni, Kepler A. Carvalho, Roni C. Fernandes, Marjo D. \\ Perez
}

Division of Urology, School of Medicine, Santa Casa de Sao Paulo, Sao Paulo, SP, Brazil

\begin{abstract}
Purpose: Several studies have documented high incidence of urinary lithiasis after jejunoileal by-pass. Roux-en-y gastric bypass surgery (RYGB) is currently the most common bariatric procedure. Because of its difficult for absorption, RYGB has a potential risk to increase the incidence of lithiasis. This study was conducted in order to test the hypothesis that RYGB increases the incidence urolithiasis after $50 \%$ of excessive weight loss.

Materials and Methods: We performed a retrospective cohort study to evaluate 58 patients who underwent RYGB at the Obesity Service at Santa Casa de Misericordia de Sao Paulo, between 2000 and 2005, with minimum follow-up of 10 and maximum of 72 months, after the procedure.

Results: Forty-five (77.6\%) patients had $\geq 50 \%$ loss of weight excess. There was no difference between the frequency of urolithiasis before and after the procedure, and nephrolithiasis was observed after surgery in only one patient, however this had been detected before the procedure.

Conclusion: In the period studied, RYGB does not seem to affect the incidence of urolithiasis after weight reduction. This may be due to its smaller malabsorptive component as compared with jejunoileal "by-pass", thereby possibly not significantly influencing the oxalate metabolism.
\end{abstract}

Key words: lithiasis; urolithiasis; obesity; bariatric surgery; gastric bypass

Int Braz J Urol. 2009; 35: 432-5

\section{INTRODUCTION}

Several studies have documented the association of malabsorption and surgery for morbid obesity and urinary lithiasis (1-3). High levels of oxalate salts in urine have also been demonstrated after mixed surgeries for weight loss (those that involve malabsorptive and restrictive components), such as biliopancreatic diversion (4). The mechanism of this disturbance is explained by lipid malabsorption leading to binding between fatty acids that are not absorbed to calcium from the intestinal lumen, leaving free the oxalate that comes from the diet. This increases the levels of oxalate in the urine and consequently facilitates the development of urinary lithiasis.

The Roux-en-Y gastric bypass (RYGB), also known as Fobi-Capella surgery, is currently considered the gold standard in bariatric surgery. It is the most frequently applied technique in Brazil and in the United States (5). Even though this technique results in malabsorption, there has been a paucity of reports on metabolic complications evolving oxalate and consequent predisposition to urolithiasis that could potentially occur after this procedure. On the other hand, other complications of this surgery have been documented, such as deficiency of vitamin 
B12, calcium and iron (1). The aim of the present study was to evaluate the association of RYGB and urolithiasis.

\section{MATERIALS AND METHODS}

We have conducted a retrospective cohort study evaluating 58 randomly selected morbid obese patients who underwent RYGB surgery at the Division of Bariatric Surgery of Santa Casa of Sao Paulo, between 2000 and 2005, with follow-up ranging from 10 to 72 months. Data were reviewed from medical records, having an ultrasonography of the urinary tract before and after the procedure, which was considered an inclusion criteria. Ultrasonographies had been performed before the surgery, six months, and yearly post surgery.

At the Division of Bariatric Surgery of Santa Casa of Sao Paulo a standard RYGB is generally performed. It consists of an average $100 \mathrm{~cm}$ length Roux limb (maximum of $150 \mathrm{~cm}$ ) - the malabsorptive component - associated with a reduction gastroplasty, building an approximately $50 \mathrm{cc}$ reservoir - the restrictive component. This surgery differs from malabsorptive procedures like JIB (jejunoileal bypass) in which only about $35 \mathrm{~cm}$ of normally absorptive small intestine was retained in the absorptive stream, compared with the normal length of approximately 7 meters, and in this case, there was no gastroplasty.
In the early postoperative period, patients were instructed to have a hypocaloric, hypolipidic and hyperproteic diet without sacarosis, consisting of a total of 300 calories a day. After weight loss, they were allowed to have an up to 1,500 calories/day diet. No oxalate restrictions were recommended.

Statistical analysis was performed with SPSS 10.0 (Statistic Package for Social Science) program. Statistic tests for dependent variables were used, as the same individuals were compared before and after the procedure. For continuous data, we used Student's-ttest, and for the dependent variables, the McNemar test. Our cut-off point of type I error was $\leq 0.05$.

\section{RESULTS}

The medium \pm standard error age was 39.3 \pm 10.8 years (range from 19 to 63 years). There were a total of $48(83 \%)$ women and $10(17 \%)$ men. In forty-five $(77.6 \%)$ patients, a weight-loss equal to or higher than $50 \%$ was achieved. When comparing pre- vs. postoperative prevalence of diabetes $(19.0 \%$ vs. $1.7 \%, \mathrm{p}<0.001)$ and hypertension $(60.0 \%$ vs. $20.0 \%, \mathrm{p}=0.02)$, a significant reduction was observed (Table-1).

There were no differences in the frequency of urolithiasis before and after surgery; urolithiasis was found in only one patient at the pre and postoperative period. This patient was an asymptomatic thirty-eight

Table 1 - Social and anthropometric information before and after excessive weight loss.

\begin{tabular}{lccc}
\hline & Variables & Weight Loss & p Value \\
\hline Social & Gender & $\mathbf{N}(\%)$ & \\
& Race & $46(79.3)$ Female & \\
& & $40(69)$ Caucasian & \\
Anthropometric & Weight before & Mean \pm SD, Minimum-Maximum & $<0.001$ \\
& Weight after & $130 \pm 20.9(83.5-176)(\mathrm{Kg})$ & \\
& Height & $82.7 \pm 16.2(42.8-131)(\mathrm{Kg})$ & $<0.001$ \\
& BMI before & $1.64 \pm 0.1(1.49-1.85)(\mathrm{m})$ & \\
\hline
\end{tabular}

$B M I=$ Body mass Index. 
year old woman with a $1 \mathrm{~cm}$ stone in the left inferior calyceal group. After a 42-month postoperative follow-up, the stone remained unchanged.

\section{COMMENTS}

Our data was similar to literature as regards the amount of weight loss (77.6\%) and in reducing hypertension and diabetes after Roux-en-y gastric bypass (6).

Despite the efficacy of malabsorptives surgeries in promoting weight loss (6), their use has been drastically reduced due to adverse effects (1-3,7-9). Several studies of urologic sequelae of jejunoileal bypass showed an association with stone formation, with varying frequencies. Annuk et al. (1) found an incidence of $39.3 \%$ after a few months to 19 years, the median being 5 years. In our study, the patients were followed for 10 to 72 months, with a median of 3 years and 6 months. Other authors have demonstrated reduction in oxalate excretion after the reversion of this surgery: Dhar et al. monitored 4 women who underwent jejunoileal bypass reversion for refractory renal calculosis and observed a mean reduction of oxalate excretion in 24 hours from 112.5 to 33.75 mg. However, citrate excretion continued, ranging 215 - $248 \mathrm{mg}$ with a mean of $226.5 \mathrm{mg}$ in 24 hours. The trend of urinary stone formation in that study was only reverted after urine alkalinization. Dean et al. (3) studied 43 patients who underwent JIB reversion due to metabolic disturbances, for different reasons, and in 9 patients, surgery was required due to nephrolithiasis. In all of these individuals, the authors noted reversion of stone arrangement propensity with a mean follow up of 77.7 months. These authors showed the significant association between bariatric malabsorptive surgeries and development of urolithiasis.

In mix surgeries, that include both restrictive and malabsorptive components, recent data suggest an association between the procedure and the development of urolithiasis. In a paper published in 2005, the authors demonstrated an increase of oxalate in urine after biliopancreatic deflection. This technique involves a common channel of the ileum of 50 to 100 $\mathrm{cm}$, which leads to a great malabsorptive effect, associated with a partial gastrectomy and a remaining a pouch of $200 \mathrm{~mL}$ (6). In this study, Palomar et al. (4) followed 35 morbidly obese patients and reported a reduction of urinary calcium and citrate associated with increased 24-hours urine oxalate, but they did not report on the incidence of urolithiasis during the study.

Nelson et al. (10) studied 21 patients with nephrolithiasis after RYGB surgery, and 14 of them had no previous history. In 20 of the patients, there was an increase of oxalate excretion. However, a limitation was that the study was not prospective and only post operative stone incidence in patients submitted to a very long limb RYGB could be evaluated $(8 / 188$, $4 \%$ ). This is a technical variation of RYGB with a common channel of the ileum of $<125 \mathrm{~cm}$, which leads to great malabsorptive effect when compared to standard surgery. Because they had not included all patients who had undergone standard RYGB in their department, they were unable to calculate the incidence of stone formation after standard RYGB. Furthermore, no imaging studies had been performed prior to the procedure, raising the possibility of preoperative stone occurrence..

Other authors (5) have also demonstrated an increase in oxalate excretion $6(0.32 \mathrm{mM} / 24 \mathrm{~h})$ and $12(0.74 \mathrm{mM} / 24 \mathrm{~h})$ months after surgery $(\mathrm{p}<0.05)$. A recent study (11) showed that patients who preoperatively had stones, there were high levels of oxalate in the urine, sufficient for leading to the development of renal failure after RYGB surgery; this finding suggests that attention to this point is needed in the postoperative management of these patients.

In our study, we did not find increased urolithiasis development after RYGB surgery after a medium 42-month follow-up. This could be explained by the minor malabsorptive component promoted by mixed surgeries. The absence of steatorrhea in our patients supports this conjecture. However, we did not study metabolic patterns, so it is not possible to ascertain whether there were alterations in oxalate metabolism in these patients.

It is known that hyperproteic diet can improve renal stone formation due to acidosis, reabsorptive hipercalciuria and urate elevation. However, in malabsorptive surgeries, like jejunoileal "bypass", the diet recommendations are similar to those after Roux-enY gastric bypass, so these influences are controlled. 
Additionally, some hypertensive patients had been using a thiazidic diuretic that could also have reduced renal stone formation. However, doses were reduced postoperatively due to a better hypertension control.

None of the patients had used citrate, but calcium supplements were routinely prescribed due to duodenal bypass, as this is the most important calcium absorptive intestinal segment. The dose used was 162 mg per day and was increased or reduced according to plasma levels. Calcium use could reduce oxalate absorption and consequently reduce stone formation, however this dosage is probably too low to cause any interference.

\section{CONCLUSIONS}

The potential association between RYGB and the incidence of urinary lithiasis has not been routinely studied. The present study did not show changes in occurrence of urolithiasis after this surgery. A prospective follow-up study with a larger number of patients, metabolic evaluation and strict control of food and calcium replacement is necessary to conclusively determine whether or not urolithiasis is a complication of RYGB surgery.

\section{CONFLICT OF INTEREST}

None declared.

\section{REFERENCES}

1. Annuk M, Backman U, Holmgren K, Vessby B: Urinary calculi and jejunoileal bypass operation. A long-term follow-up. Scand J Urol Nephrol. 1998; 32: $177-80$.

2. Dhar NB, Grundfest S, Jones JS, Streem SB: Jejunoileal bypass reversal: effect on renal function, metabolic parameters and stone formation. J Urol. 2005; 174: 1844-6; discussion 1846.

3. Dean P, Joshi S, Kaminski DL: Long-term outcome of reversal of small intestinal bypass operations. Am J Surg. 1990; 159: 118-23; discussion 123-4.

4. Palomar R, Fernández-Fresnedo G, Domínguez-Diez A, López-Deogracias M, Olmedo F, Martín de Fran- cisco AL, et al.: Effects of weight loss after biliopancreatic diversion on metabolism and cardiovascular profile. Obes Surg. 2005; 15: 794-8.

5. Sinha MK, Collazo-Clavell ML, Rule A, Milliner DS, Nelson W, Sarr MG, et al.: Hyperoxaluric nephrolithiasis is a complication of Roux-en-Y gastric bypass surgery. Kidney Int. 2007; 72: 100-7.

6. Schirmer BD: Obesidade Mórbida. In: Townsend CM, Beauchamp ED, Evers BM, Mattox KL (eds.), Sabiston Tratado de Cirurgia. A Base Biológica da Prática Cirúgica Moderna. Rio de Janeiro, Elsevier. 2005; pp. 357-99.

7. Mango VL, Frishman WH: Physiologic, psychologic, and metabolic consequences of bariatric surgery. Cardiol Rev. 2006; 14: 232-7.

8. Malinowski SS: Nutritional and metabolic complications of bariatric surgery. Am J Med Sci. 2006; 331: 219-25.

9. Ocón Bretón J, Pérez Naranjo S, Gimeno Laborda S, Benito Ruesca P, García Hernández R: Effectiveness and complications of bariatric surgery in the treatment of morbid obesity. Nutr Hosp. 2005; 20: 409-14.

10. Nelson WK, Houghton SG, Milliner DS, Lieske JC, Sarr MG: Enteric hyperoxaluria, nephrolithiasis, and oxalate nephropathy: potentially serious and unappreciated complications of Roux-en-Y gastric bypass. Surg Obes Relat Dis. 2005; 1: 481-5.

11. Asplin JR, Coe FL: Hyperoxaluria in kidney stone formers treated with modern bariatric surgery. J Urol. 2007; 177: 565-9.

Accepted after revision:

March 12, 2009

\section{Correspondence address:}

Dr. André Matos

Rua Martinico Prado, 106

São Paulo, SP, 01224-010, Brazil

E-mail: andrecostamatos@yahoo.com.br 